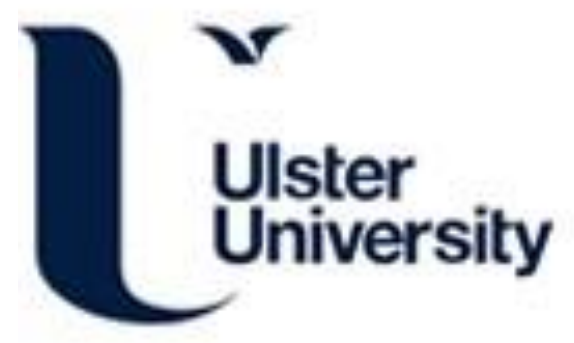

\title{
Design of Composite Microneedle Sensor Systems for the Measurement of Transdermal pH
}

Hegarty, C., McConville, A., Mc Glynn, R., Mariotti, D., \& Davis, J. (2019). Design of Composite Microneedle Sensor Systems for the Measurement of Transdermal pH. Materials Chemistry and Physics, 227, 340-346. https://doi.org/10.1016/j.matchemphys.2019.01.052

Link to publication record in Ulster University Research Portal

\section{Published in:}

Materials Chemistry and Physics

Publication Status:

Published (in print/issue): 01/04/2019

DOI:

10.1016/j.matchemphys.2019.01.052

\section{Document Version}

Author Accepted version

\section{General rights}

Copyright for the publications made accessible via Ulster University's Research Portal is retained by the author(s) and / or other copyright owners and it is a condition of accessing these publications that users recognise and abide by the legal requirements associated with these rights.

\section{Take down policy}

The Research Portal is Ulster University's institutional repository that provides access to Ulster's research outputs. Every effort has been made to ensure that content in the Research Portal does not infringe any person's rights, or applicable UK laws. If you discover content in the Research Portal that you believe breaches copyright or violates any law, please contact pure-support@ulster.ac.uk. 


\title{
Design of Composite Microneedle Sensor Systems for the Measurement of Transdermal pH
}

\author{
Catherine Hegarty ${ }^{1}$, Aaron McConville ${ }^{1}$, Ruairi J. McGlynn ${ }^{1}$, Davide Mariotti ${ }^{1}$ and James Davis ${ }^{1 *}$ \\ ${ }^{1}$ School of Engineering, Ulster University, Jordanstown, Northern Ireland, BT37 0QB, UK
}

\begin{abstract}
Carbon loaded polystyrene microneedle patches have been prepared using silicone micromoulding techniques and the ability of the needles to serve as viable transdermal sensors has been evaluated. The population of quinone groups at the interface of the embedded carbon nanoparticles was increased through anodisation and their $\mathrm{pH}$ dependent redox transitions exploited as the basis of a reagentless $\mathrm{pH}$ sensor. The peak position of the quinone oxidation process was found to shift in accordance with Nernstian behaviour and the influence of penetration depth on response has been investigated. The analytical applicability of the microneedle electrode patch was critically evaluated through using tomato skin as model transdermal skin mimic. Despite the increased complexity of the matrix, the microneedle sensor response was found to compare favourably with conventional/commercial pH probes.
\end{abstract}

\section{Keywords}

Composite; $\mathrm{pH}$; sensor; microneedle; quinones; transdermal

\footnotetext{
${ }^{1}$ To whom correspondence should be addressed. T: +44(0)28 903 66407; E: james.davis@ulster.ac.uk
} 


\subsection{Introduction}

Interest in the development of microneedle (MN) technologies for drug delivery applications has seen a near exponential rise in recent years offering significant advances over conventional treatment options [1-3]. The increasing accessibility of silicone templates has also spurred considerable attention allowing rapid production of prototypes within conventional laboratories [4-8]. These micro-moulding techniques can also be used to produce $\mathrm{MN}$ arrays for diagnostic purposes allowing the extraction of interstitial fluid [9-12] for secondary in vitro analyses or facilitating the design of a new field of wearable sensors enabling the in situ measurement of sweat or disease biomarkers [6-8,12-16]. The potential for integrating MN sensors within closed loop therapies has also garnered considerable interest in recent years [17]. While early MN designs were typically based on micromachining and photolithographic methods, the preparation of polymeric MN arrays has opened up new avenues of sensor design[1-3]. The deposition of metallic layers through sputtering or evaporation onto polymer structures is a common approach in sensor design [6-8] but the field has developed considerably and composite systems involving conductive/catalytic particles are beginning to emerge $[5,15,16,18]$. The incorporation of carbon nanoparticles within an inert/biocompatible polymer could, in principle, open up a wide spectrum of electroanalytical sensing opportunities given the wide sensing window available at the interfacial carbon and its ease of modification. The aim of this communication seeks to investigate the production of such a system and its modification to yield functionalities sensitive to $\mathrm{pH}$.

Carbon composite electrodes are well established as viable diagnostic substrates and there is an extensive literature base on the development of disposable screen printed carbon electrodes (SPEs) for the measurement of a wide range of disease biomarkers [19-21]. In contrast, the adaption of carbon formulations to microneedle formats has yet to be explored, but they could offer significant advantages over conventional printed systems. The latter are typically positioned to operate on the basis of pin prick sampling of capillary blood which can be painful and brings issues of patient compliance. Microneedle patches have long been proffered as a minimally invasive approach to treatment with their near painless insertion into the epidermal layers presenting only a pressing sensation during application [1-3]. This is based on the concept that the needle penetration is limited to the upper layers of the skin and fail to trigger the underlying dermal nerve network. It is little surprise therefore that such systems offer an opportunity to 
revolutionise vaccination programmes for children where needle phobia can dramatically reduce participation [3]. Given that the proposed sensing MN arrays possess similar dimensions, it could be envisaged that they would similarly benefit from the pain-free administration and could be a step-change in diagnostic screening.

Rather than being a simple film coating a pre-patterned MN structure, the composite system approach utilises a micromoulding system in which a mixture of carbon nanoparticles are initially dispersed in a polystyrene/cyclohexane solution and then cast into a silicone template. As the cyclohexane solvent evaporates a solid composite results in which the carbon nanoparticles are homogeneously distributed within the polystyrene binder. In this case, a single solid phase is achieved in which the carbon nanoparticles provide a conductive path from needle tip to base plate. Electrical connection to the microneedles is achieved through the use of a carbon fiber stub which is suspended in the outer side of the base plate during the period in which the solvent evaporates. The outer side of the base plate is then coated in an enamel dielectric to ensure that only the face containing the needles serves as the electrode-solution interface and therein defines the geometrical surface area.

The basic composition of the proposed measurement system is highlighted in Figure 1. Carbon loaded polystyrene serves as the main component of both the working and reference electrodes with the polymer providing the mechanical integrity required to hold the particles in place and to puncture skin. In addition, silver was incorporated into the reference patch (either as discrete nanoparticles or as an electrodeposited layer) to serve as a silver-silver chloride pseudo reference and mirrors the approach taken by Sharma and colleagues [7]. 


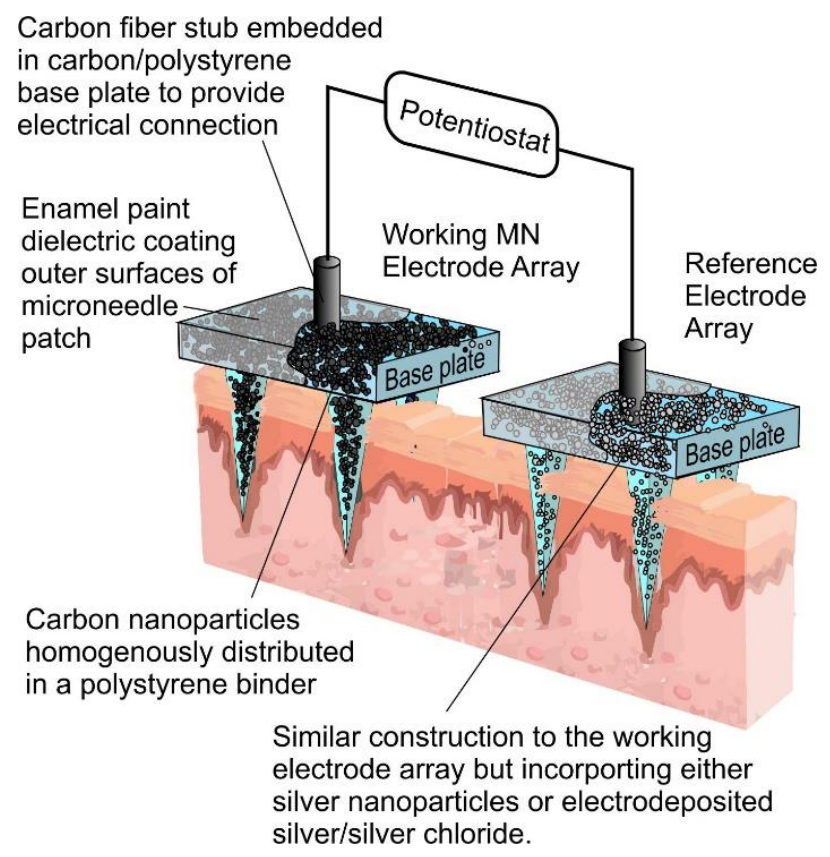

Figure 1. Proposed carbon loaded polystyrene microneedle sensing array configuration

While carbon has a wide potential window for voltammetric applications, basal plane features tend to predominate on the particles and, as such, large overpotentials are often required which can limit selectivity and sensitivity of the measurements. Exfoliation of the interfacial particles through either chemical or electrochemical means can increase both the proportion of edge plane sites and oxygen functionality[22-24]. This has the effect of improving surface wetting but can also dramatically improve the electron transfer kinetics for some species and therein the electroanalytical performance. The ability of electrochemical anodisation processes to alter the chemical functionality of carbon electrode surfaces has been confirmed through XPS analysis where a variety of oxygen functional groups are incorporated directly in to the carbon structure[22,23]. In the present investigation, the authors exploited the electrochemical anodisation of the $\mathrm{MN}$ array as a means of increasing the population of endogenous quinone functionalities (Figure 2) whose inherent $\mathrm{pH}$ dependent redox activity could be used as an indirect means of measuring $\mathrm{pH}$. Quinones are known to exhibit proton dependent electrochemical behaviour and they have formed the basis of a number of $\mathrm{pH}$ sensors whereby the peak position of the oxidation processes have been shown to shift with changes in $\mathrm{pH}$ [25-27]. In this case, the quinones are locked within the carbon particles embedded in the microneedle structures and thus provide a simple route to the production of reagentless sensors. 


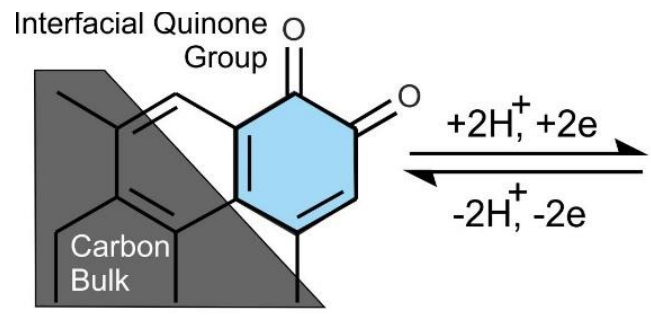

(I)

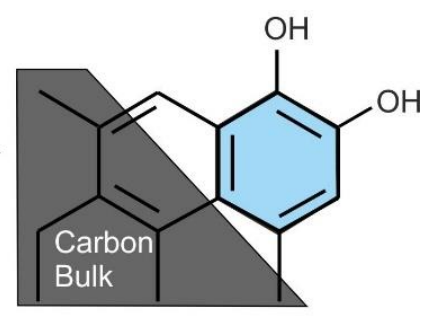

(II)

Figure 2. Redox transformations of the quinone groups associated with the carbon particles embedded with the microneedle structure.

The electroanalytical performance of the carbon loaded microneedles was assessed through examining the $\mathrm{pH}$ of tomato skin and its underlying flesh whereby the latter provides a model transdermal substrate easing the processes of validation by providing opportunities to excise the skin for electron microscopy and removing ethical issues of applying the sensing assembly shown in Figure 1.

\subsection{Experimental Details}

Materials and Instrumentation: All chemicals were obtained from Sigma-Aldrich, were the highest grade available and were used without further purification. Britton Robinson buffers (acetic, boric, and phosphoric acids, each at a concentration of $0.04 \mathrm{M}$ and adjusted to the appropriate $\mathrm{pH}$ through the addition of sodium hydroxide) were used throughout unless otherwise specified. Electrochemical analysis was carried out using a micro Autolab (Type III) computer controlled potentiostat (Eco-Chemie) with either a standard three-electrode configuration in which the microneedle patch was used as the working electrode with platinum and a $\mathrm{Ag} \mid \mathrm{AgCl}$ half cell (3M NaCl, BAS Technicol UK) acting as counter and reference respectively. Later investigations exploited a two electrode system in which a silver loaded microneedle patch was used as the combined reference-counter electrode. While little current passes through the reference in three electrode systems and thereby acts to preserve the reference potential, in two electrode systems this is not the case and the silver modified MN patch must therefore act as both counter and pseudo reference. The two electrode approach can greatly simplify the electrode cell design but can come at a cost of reference stability as consequence of current passage and this is aspect is considered later in the text. 
All measurements were conducted at $22^{\circ} \mathrm{C} \pm 2^{\circ} \mathrm{C}$. X-ray microtomography was carried out using a SkyScan 1275 Micro-CT system (Bruker Corporation, USA), with a rotation angle increment of 0.20 degrees and an average of 3 frames per increment. Reconstruction and analysis were carried out using the accompanying NRecon and CTVox visualisation software respectively. Conductivity measurements were obtained using a 2461 series SourceMeter ${ }^{\circledR}$ (Keithley) 4 point probe. The conductivity of an unmodified carbon-polystyrene microneedle patch was found to be $1575.2 \mathrm{~S} / \mathrm{m}$ $\pm 96 \mathrm{~S} / \mathrm{m}(\mathrm{N}=5)$.

A Kratos Axis Ultra DLD Spectrometer was used to quantify surface composition and acquire X-ray photoelectron spectroscopy (XPS) spectra. Spectra were analysed using monochromated Al Ka X-rays ( $\mathrm{hv}=1486.6$ electron volts $(\mathrm{eV})$ ) operating at $15 \mathrm{kV}$ and $10 \mathrm{~mA}$ (150W). During analysis, a hybrid lens mode was used (electrostatic and magnetic) with a $300 \mu \mathrm{m}$ $x 700 \mu \mathrm{m}$ analysis area and a take-off angle (TOA) of $90^{\circ}$ with respect to the sample surface. Wide energy survey scans (WESS) were collected across a range of -5 to $1200 \mathrm{eV}$ binding energy (BE), with a pass energy of $160 \mathrm{eV}$ and step size of $1 \mathrm{eV}$. High-resolution spectra were collected with a pass energy of $20 \mathrm{eV}$ with a $0.05 \mathrm{eV}$ step size, a scan width of $25 \mathrm{eV}$, a dwell time of 150 milliseconds and at least 3 sweeps to reduce the signal noise. A Kratos charge neutraliser system with a filament current of $1.8 \mathrm{~A}$ and a charge balance of $3.6 \mathrm{~V}$ and a filament bias of $1.3 \mathrm{~V}$ was used for all samples. Charging effects on the BE positions were adjusted by setting the lowest BE for the $\mathrm{C} 1 \mathrm{~s}$ spectral envelope to $284.8 \mathrm{eV}$, which is commonly accepted as adventitious carbon surface contamination. Three measurements were analysed per sample, with a Shirley background subtracted from each XPS spectra. The peak areas of the most intense spectral lines for each elemental species were used to determine the percentage atomic concentration. Peak fitting of high-resolution spectra was carried out using Casa XPS software.

Raman spectra were obtained using a Horiba LabRAM 300 spectrometer employing a HeNe laser (632 nm, 50 X optical lens, spot approx. $30 \mu \mathrm{m}, 10 \%$ filter, laser power $2.59 \mathrm{~W}$ (25.9 * $10 \%))$. The carbon-polystyrene composite samples were analysed over $100-3500 \mathrm{~cm}^{-1}$ conduced in 5 slices (energy per slice $38.85 \mathrm{~J}$ ) to cover the range with 5 spots per sample.

Preparation of Microneedles: Carbon nanoparticles (Sigma UK) were combined with polystyrene powder $(100,000$, Sigma) in a ratio of $1: 1$ by weight, dissolved in cyclohexanone and stirred until a homogenous solution was obtained (typically 2 hours). The solution was then cast into silicone 
microneedle templates (Micropoint ${ }^{\circledR}$ ) and a carbon fibre stub was placed into the base plate section to facilitate electrical connection. The dimensions of the silicone templates were 200 (b) $x$ $500(p) \times 700(h)$ micron covering a $10 \times 10$ needle array. The templates were placed in a vacuum at $30^{\circ} \mathrm{C}$ and once the pressure had increased to $1000 \mathrm{mbar}$ the air was released again (this was to draw the homogenous solution to the tips of the silicone cast ensuring sharp microneedle production). If required after vacuuming, the templates were topped up with more solution preferably overflowing the cast to allow for solvent evaporation. The solvent was left to evaporate at room temperature (over $48 \mathrm{hrs}$ ) whereupon the needles could be removed from the patch. The baseplate and non-needle surfaces were coated with enamel (6 hour drying period) to serve as a dielectric and define the geometric electrode area.

Preparation of the Silver-Loaded Microneedle Reference: A layer of silver was first electrochemically deposited onto the carbon surface. This was achieved using chronoamperometry in which the electrode was held at - $1 \mathrm{~V}$ for 60 seconds in a solution containing silver nitrate $\left(0.01 \mathrm{M}, 0.1 \mathrm{M} \mathrm{HNO}_{3}\right)$. The electrode was removed, rinsed and placed in $0.1 \mathrm{M} \mathrm{KCl}$ and a single cyclic voltammogram run $(-0.8 \mathrm{~V}$ to $+0.8 \mathrm{~V}$ at $50 \mathrm{mV} / \mathrm{s})$ in order to oxidise the electrodeposited silver and create a layer of silver chloride. The presence of silver on the microneedle structures was confirmed through SEM/EDX and XPS analysis. Images of the electrodeposit and accompanying EDX profile and high resolution Ag 3d XPS data are supplied in the supporting information file. The percentage atomic concentration of silver on the surface of the reference $\mathrm{MN}$ array was found to be $4.8 \%$. The resulting silver-silver chloride pseudo reference was characterised using conventional potentiometric techniques in varying chloride concentrations with a $-58.9 \mathrm{mV}$ per decade chloride ion response consistent with Nernstian behaviour. 


\subsection{Results and Discussion}

Cyclic voltammograms detailing the response of the carbon loaded $\mathrm{MN}$ assembly towards ferrocyanide $(2 \mathrm{mM}, 0.1 \mathrm{M} \mathrm{KCl}, 50 \mathrm{mV} / \mathrm{s})$ before and after anodisation are compared in Figure $3 \mathrm{~A}$. It can be seen that there is a significant improvement in the $\mathrm{MN}$ response post anodisation with much greater definition of the peak processes and smaller peak separation $\left(\Delta \mathrm{E}_{\mathrm{p}}=360 \mathrm{mV}\right)$. The latter is still significantly larger than that expected for the ferro-ferricyanide couple and can be attributed in part to the composite nature of the $\mathrm{MN}$ array where internal resistance is inevitable [5]. The poor response of the unmodified MN to ferrocyanide is consistent with previous studies where electrodes composed predominantly of basal plane surfaces with few surface defects similarly exhibit slow electron transfer kinetics[22-24]. In such studies, the response to ruthenium hexamine was found to be superior to the ferrocyanide and this was also observed in this investigation (Figure 2B) where there is marginal difference between the unmodified and anodised surfaces [24].

While it could be envisaged that increasing the carbon loading would further improve the electroanalytical performance through decreasing the internal resistance, it is important to acknowledge that a compromise is required in the construction of the $\mathrm{MN}$ array. The conductive elements are the carbon nanoparticles and while there must be a sufficient density to enable conduction, they possess no inherent adhesive qualities. The polystyrene must therefore serve to bind the particles but also provide the structural integrity that will confer mechanical strength to penetrate skin surfaces. It has been found that decreasing the amount of polystyrene below $50 \%$, whist enabling the production of MN, leads to a more granular morphology and inherently brittle needle structure. 

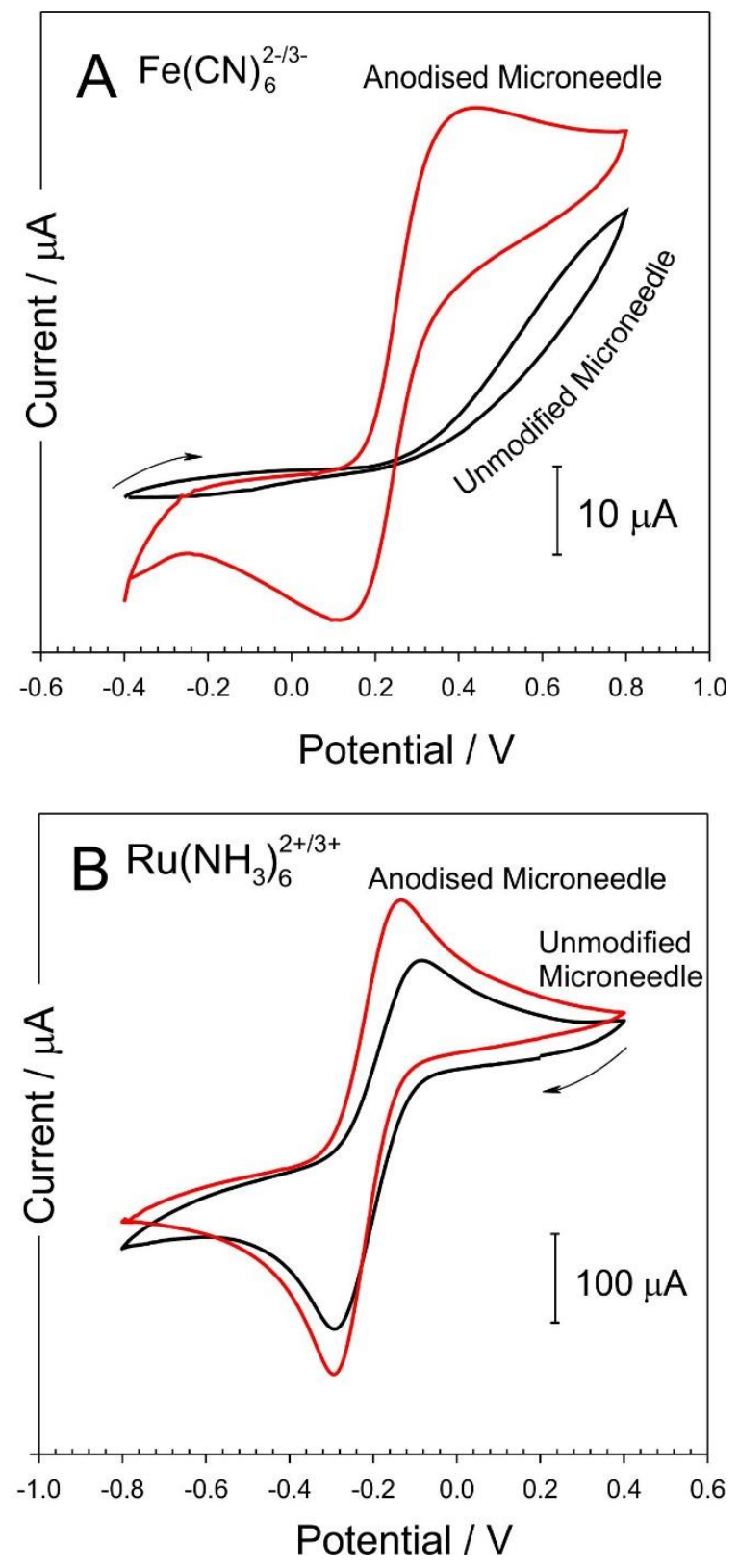

Figure 3. Cyclic voltammograms detailing the response of the carbon loaded polystyrene microneedle patch (single $\mathrm{MN}$ patch) before and after anodisation towards $2 \mathrm{mM}$ ferrocyanide $(\mathrm{A})$ and $2 \mathrm{mM}$ ruthenium hexamine (B). Scan rate in both instances: $50 \mathrm{mV} / \mathrm{s}$.

The effect of anodisation on the carbon-polystyrene composite was also investigated using Raman spectroscopy. The spectra detailed in Figure 4 were normalised to G-band maximum intensity and it can be seen that the D-band (hence disorder in the $\mathrm{sp}^{2}$ lattice) increases slightly as the anodisation time is increased. The 2-D $\left(\mathrm{G}^{\prime}\right)$ band appears to decrease in intensity. The increase in 
disorder is consistent with previous studies examining the anodisation of carbon fibre [23] but it is also apparent from the spectra that there is a loss of carbon from the electrode-solution interface. This can be attributed to the nature of the carbon forming the composite structure where it is inevitable that there will be loosely bound carbon particles at the interface. The subsequent anodisation process and the electrolytic evolution of oxygen at the carbon interface will lead to the particles becoming dislodged and thereby reveal more of the polystyrene binder. This is corroborated in the spectra in Figure 4 where bands associated with the polystyrene are more prominent at longer anodisation times. It is also noteworthy that prevalence of $\mathrm{OH}$ functionality is also evident and increase with time which is again consistent with the proposed strategy outlined in Figure 2 and supports the improved electron transfer kinetics observed in Figure $3 \mathrm{~A}$.

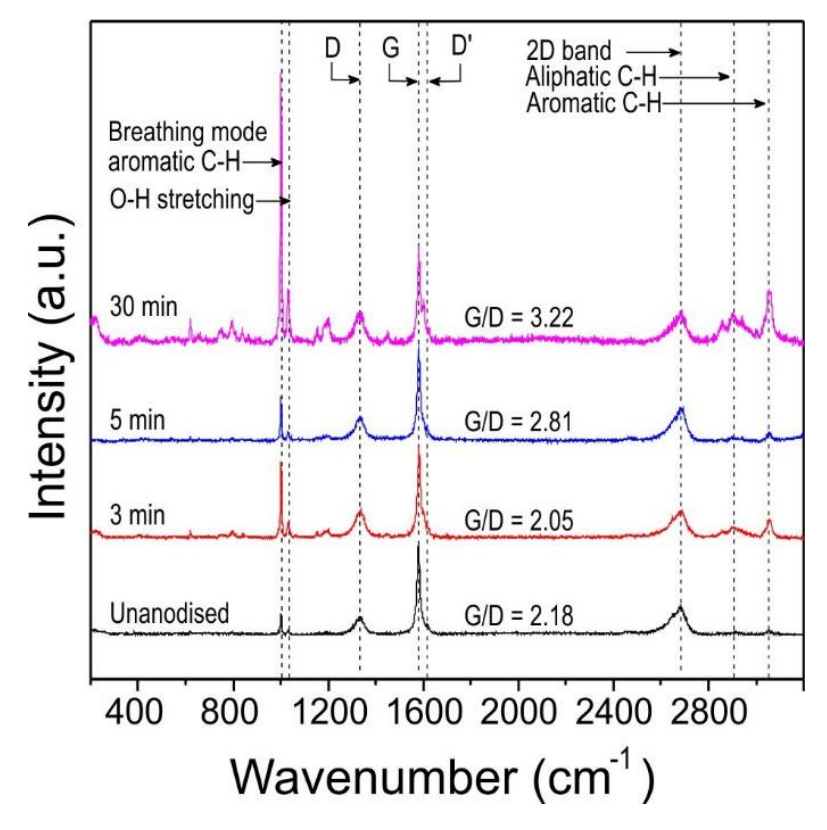

Figure 4. Raman spectra detailing the influence of electrochemical anodisation on the carbon-polystyrene composite surface structure.

\section{Microneedle pH Response}

The core sensing methodology relates to the imposition of an initial reducing potential that immediately induces the reduction of the quinone species (I -> II, Figure 2 ) present at the solution interface within the carbon particles. The potential of the electrode is then swept in an anodic direction whereupon the re-oxidation of the quinone groups occurs (II -> I, Figure 2). The position of the oxidation peak process is dependent on $\mathrm{pH}$ and hence its exploitation as an indirect marker 
for the latter[25-29]. Given the critical nature of ascribing the peak position, the differential nature of the waveform in square wave voltammetry was used in resolving peak processes. Square wave voltammograms detailing the response of the $\mathrm{MN}$ patch in buffers of varying $\mathrm{pH}$ are shown in Figure $5 \mathrm{~A}$. The oxidation of the surface hydroquinone groups back to the quinone is clearly observed with well defined peak profiles. Moreover, the position of the oxidation peak can be seen to shift with $\mathrm{pH}\left(\mathrm{E}_{\mathrm{pa}} / \mathrm{V}=-0.0575 \mathrm{pH}+0.4107 ; \mathrm{N}=6 \mathrm{R}^{2}=0.999\right)$ in accordance with Nernstian behaviour and is consistent with previous reports where quinones have been covalently tethered to electrode surfaces [25-28].
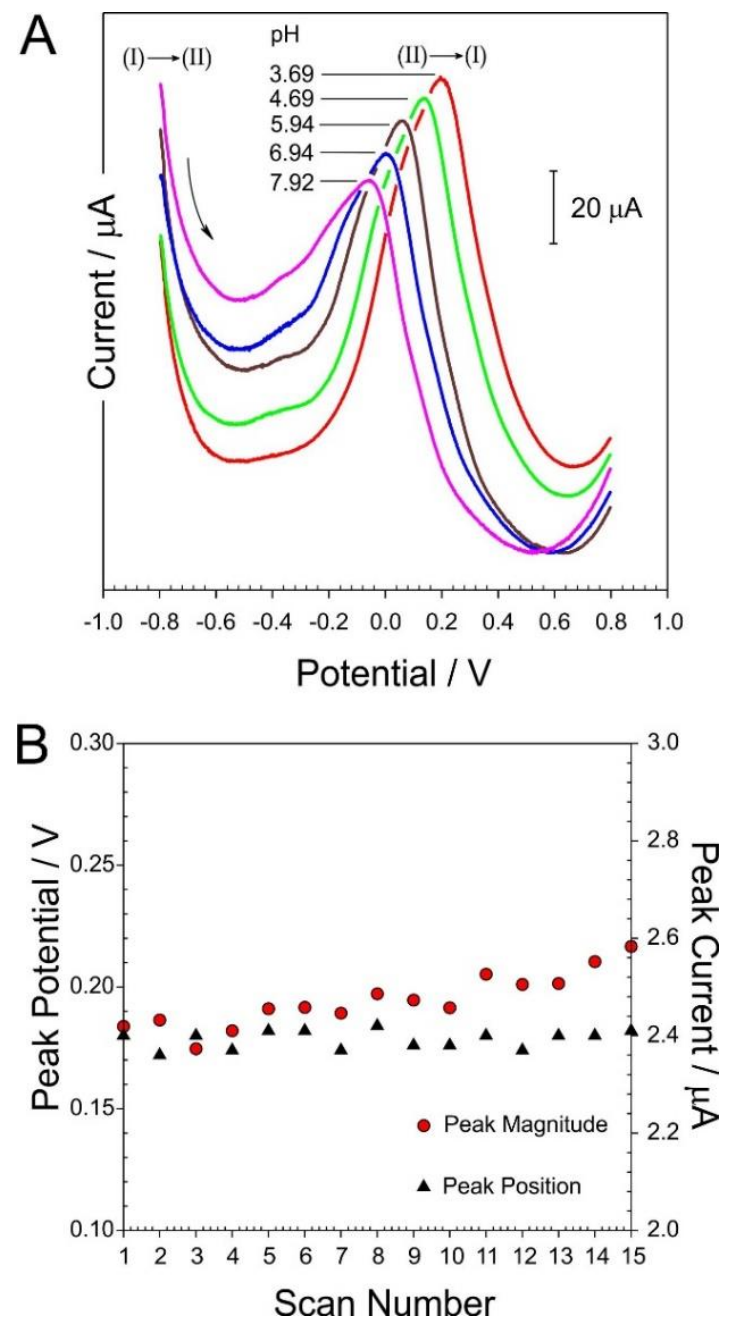

Figure 5. A) Square wave voltammograms detailing the response of a single carbon loaded polystyrene microneedle patch in Britton Robinson buffers of varying $\mathrm{pH}$. B) Effect of repetitive cycling of a microneedle patch in $\mathrm{pH} 3$ buffer. 
The stability of both the working and reference electrodes towards repetitive cycling was investigated over a total of 15 scans in $\mathrm{pH} 3$ buffer in which a dual MN system was employed. The peak potential and peak current were measured for each scan and the results are summarised in Figure 5B. The peak potential was found to exhibit minimal drift over the test duration $\left(E_{p}=0.178\right.$ $\mathrm{V} \pm 1 \mathrm{mV} ;$ Std Dev = 0.0036; $\mathrm{N}=15$ ). Had the $\mathrm{MN}$ reference electrode been unstable, a significant drift in the peak potential would have been expected. Similarly, the anodic oxidation employed in the initial treatment of the microneedle array, critical to the formation of the endogenous quinone groups indicated in Figure 2, was not found to compromise the stability of MN response little variation in the peak current observed $\left(I_{p a}=2.48 \mu \mathrm{A} \pm 0.02 \mu \mathrm{A} ; \mathrm{N}=15\right)$.

It is important to note that the focus for voltammetric $\mathrm{pH}$ sensing tends be predominantly on the magnitude of the peak potentials rather than on the current responses obtained. The current will be dependent on electrode area. Thus, increasing the penetration depth (i.e. of the needles into the skin) will likewise increase the MN surface that is in contact with electrolyte and hence increase the current. This effect was demonstrated through controlling the penetration depth achievable by the MN patch. The latter was placed into a soft cellulose filter membrane of fixed thickness) secured on a solid glass surface and thus the active surface area is controlled by the membrane thickness. Square wave voltammograms detailing the response obtained with single and dual layer membranes (corresponding to $180 \mu \mathrm{m}$ and $360 \mu \mathrm{m}$ respectively) saturated with $\mathrm{pH}$ 7 buffer are shown in Figure 6. As expected the magnitude of the peak response for the dual membrane puncture is significantly greater than that observed with the single layer. Were the microneedles to be used for the voltammetric determination of a biomarker where the current would typically be related to concentration - this would be a significant issue. In the present context however, the significance of the current is lessened to the point of simply being able to discriminate the peak process. The critical factor here is the peak position. While the peak currents for the two scenarios outlined in Figure 6 vary considerably, it is vital to note that the peak position remains constant and effectively independent of penetration depth. 


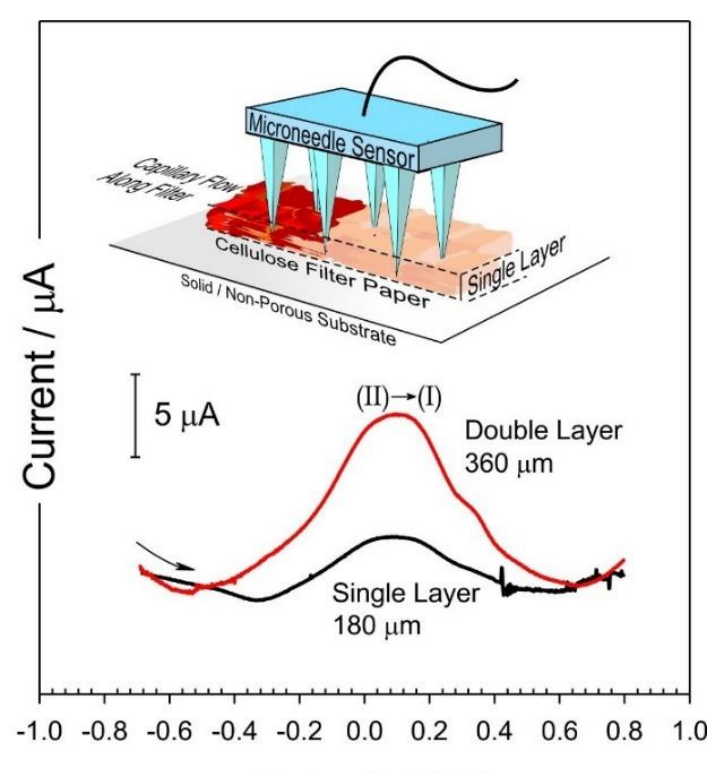

Potential / V

Figure 6. Square wave voltammograms detailing the influence of penetration depth on the peak position and current response of a single carbon loaded polystyrene microneedle sensing patch in $\mathrm{pH} 7$ buffer.

\section{Analysis of Tomato Skin}

Having negated the issue of penetration depth, the next challenge was to determine if the system could accurately measure the $\mathrm{pH}$ of the tomato flesh. Scanning electron micrographs of the carbon-polystyrene microneedles are shown in Figure 7A and their penetration through tomato skin is shown in Figure 7B and 7C. In the case of the latter - the tomato flesh was removed from the cuticle in order to highlight the piercing capability of the $M N$ structures. The web-like patterning on the skin's inner surface (Figure 7C) is an artefact of the mounting process where the cell wall structures remain strongly adherent to the cuticle. 

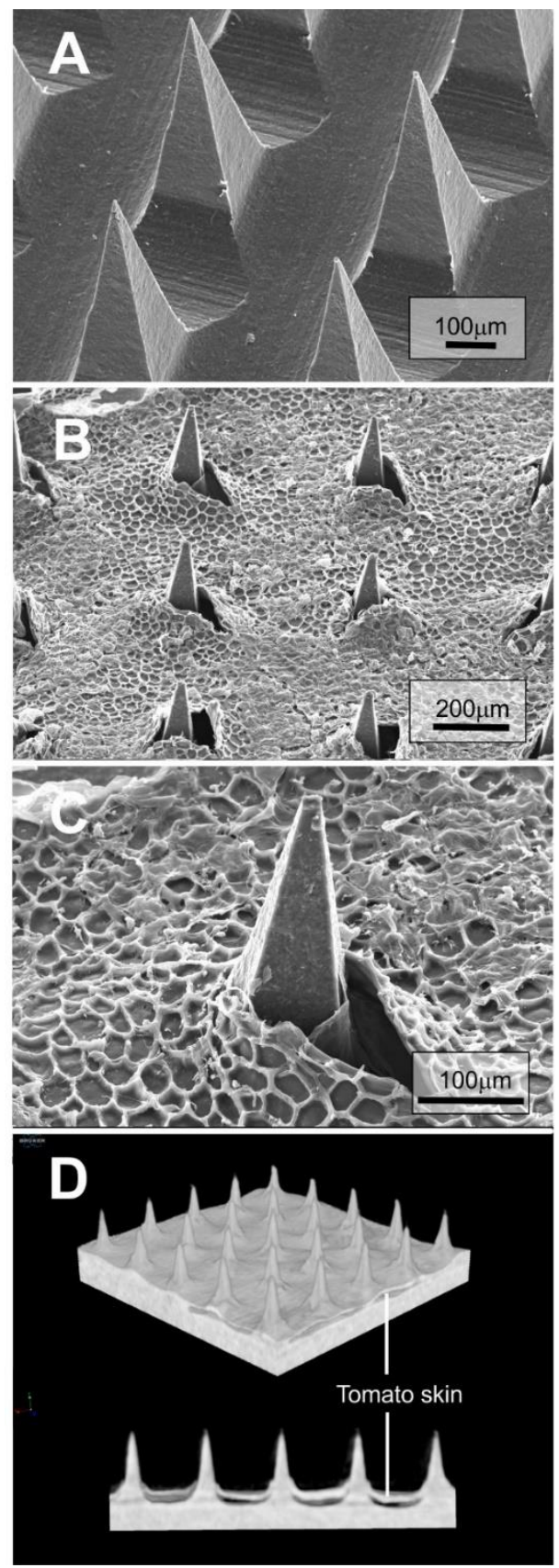

Figure 7. Scanning electron micrographs of the carbon loaded polystyrene microneedle patch before (A) and after $(B, C)$ piercing through tomato skin. $C T$ scans of the patch after puncturing the tomato skin (D).

Computerised tomography (CT) x-ray scans were also conducted of the needle post piercing and representative images are detailed in Figure 7D. Cross sectional analysis of the needle structure highlights that the needles are solid with no significant fissures or voids in the main body and it can be seen from both the SEM (Figure 7B) and the CT (Figure 7D) that the needles retain 
their integrity even after the extensive handling necessary to obtain the images. On first inspection of the SEM images, there is a suggestion that the penetration depth is relatively shallow as only the tips are visible. The CT scans however demonstrate that the needles do substantially penetrate the skin. The difference can be accounted for by the fact that the CT scans were obtained at atmospheric pressure whereas the high vacuum necessary for the preparations (Au/Pd sputtering) and the actual SEM images leads to the drying of the skin and subsequent shrinkage which raises it upwards. This can be seen in the pierced holes in the skin which are larger than the needle protruding from them. Clearly, as the needles penetrate into the skin - the hole size will increase as the needle becomes wider at the base.

The analysis of the tomato skin $\mathrm{pH}$ with the microneedle patch was conducted using two strategies. First, a dual MN system was used (as indicated in Figure 1) where the MN patches serve as the sensing and reference electrodes and, second, a conventional three electrode system was used in which the reference $\mathrm{MN}$ was replaced with a conventional $\mathrm{Ag} \mid \mathrm{AgCl}$ half cell and a Pt wire acting as the counter electrode. Both approaches were then validated through the comparing the responses with a commercial $\mathrm{HALO}^{\circledR}(\mathrm{HI}-14142)$ wireless flat $\mathrm{pH}$ electrode. A two point calibration system, akin to that used with the standard potentiometric $\mathrm{pH}$ electrode, was employed throughout. Square wave voltammograms detailing the response of the MN patch (2 electrode system) to $\mathrm{pH} 3.13$ and pH 7.22 buffers are shown in Figure 8. Broad oxidation peak processes can be seen at $+0.204 \mathrm{~V}$ and $-0.013 \mathrm{~V}$ for the $\mathrm{pH} 3.13$ and $\mathrm{pH} 7.22$ buffers respectively and are consistent with the response of the single $\mathrm{MN}$ system responses detailed in Figures 5A. The response of the 2 electrode dual $\mathrm{MN}$ and the single $\mathrm{MN}$ ( 3 electrode) systems after application to the tomato skin are detailed in Figure 8 - scans A and B respectively. 


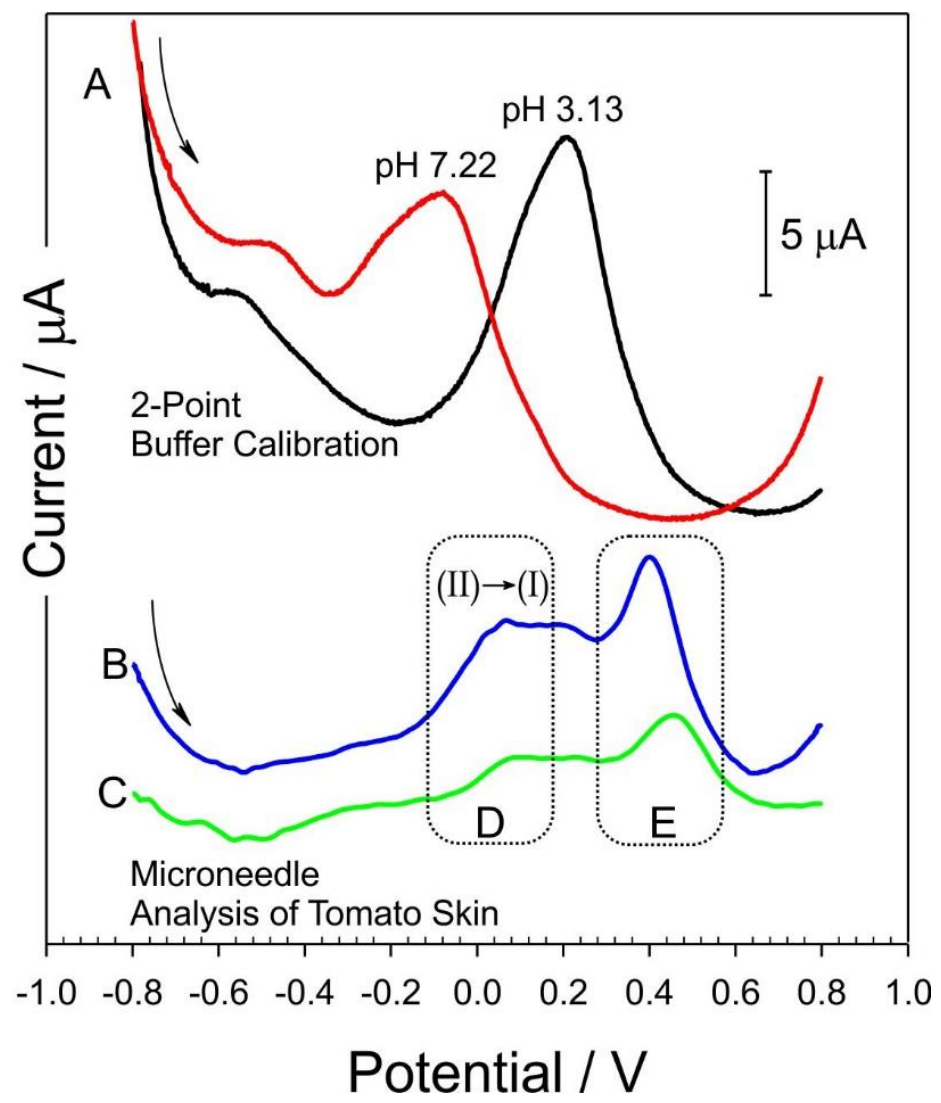

Figure 8. Square wave voltammograms highlighting (A) 2 point calibration in $\mathrm{pH} 3.13$ and pH 7.22 Britton Robinson buffer and the direct response of a single $\mathrm{MN}(\mathrm{B})$ and dual $\mathrm{MN}(\mathrm{C})$ patch system towards tomato skin. The quinone analytical region (D) and oxidation of matrix components (E) are also highlighted

There is considerable difference between the simple buffer responses and those obtained from the MNs penetrating into the tomato flesh. This can be expected given the increased complexity of the sample matrix but it is important to note that the oxidation of the embedded hydroquinone groups (II->I) is still visible and is annotated in Figure 8 (C). A second oxidation peak is also observed in the tomato samples (Figure 8 (D)) which was absent from the buffer response and is endogenous to the tomato matrix[30]. The identity of the species giving rise to the peak process is unattributed but it is noteworthy that the low oxidation potential of the embedded quinone functionalities still enables the $\mathrm{pH}$ of the underlying tomato flesh to be evaluated. The response to the single $\mathrm{MN}$ sensing system (Figure $8(\mathrm{~B})$ ) is similar in profile to that of the simpler dual MN system. The MN responses and calculated $\mathrm{pH}$ (based on the 2 point calibration using Epa $\mathrm{N}=-0.0484 \mathrm{pH}+0.3945)$ are compared with the responses measured on the commercial HALO ${ }^{\circledR}$ pH electrode in Table 1. 


\begin{tabular}{lccc} 
System & $\begin{array}{c}\text { Actual } \\
\mathrm{pH}\end{array}$ & $\begin{array}{c}\text { Calculated } \\
\mathrm{pH}\end{array}$ & $\mathrm{N}$ \\
\hline $\begin{array}{l}\text { Dual MN } \\
\text { (2 Electrode) }\end{array}$ & 4.52 & 4.77 & 5 \\
$\begin{array}{l}\text { Single MN } \\
\text { (3 Electrode) }\end{array}$ & 4.43 & 4.48 & 5 \\
\hline
\end{tabular}

Table 1. Comparison of tomato skin/flesh measured with the microneedle sensors

The three electrode system incorporating a single $\mathrm{MN}$ electrode patch compares well with the commercial system and is within $0.1 \mathrm{pH}$ and highlights the ability of the embedded quinone systems to serve as an indirect $\mathrm{pH}$ sensor. In contrast, the dual $\mathrm{MN}$ system has a larger discrepancy and it could be postulated that the difference is due to the nature of the reference electrode. In the case of the 3 electrode system, a standard $\mathrm{Ag} \mid \mathrm{AgCl}$ half cell was used which essentially maintains a constant reference potential (dictated by the internal chloride concentration). The dual system has only a pseudo reference and, while it is functionalised with a $\mathrm{Ag} \mid \mathrm{AgCl}$ layer, is nevertheless dependent on the chloride ion within the tomato flesh. Any significant deviation between the chloride concentration in the tomato flesh and that used in the calibration buffer will lead to an error. In general, however, it can be seen that the simpler dual MN system is still close to that of the actual $\mathrm{pH}$. One option that may be worthy of consideration in future designs is the incorporation of an internal reference system in which a redox species which is independent of $\mathrm{pH}$ is co-immobilised onto the electrode surface. Such systems, typically based on ferrocene or ferrocyanide, have been shown to counter the issue of variable chloride concentration [25-29] but would nevertheless add an additional layer of complexity to the fabrication process. The need for the latter would depend on the nature of the application and the level of precision required.

Microneedle systems have been employed for various sensing purposes within plant physiology [31,32] but, this is the first report highlighting the use of composite patches for voltammetric sensing. While the work detailed here has demonstrated the capability of the $\mathrm{MN}$ systems for measuring $\mathrm{pH}$, it is clear that the electroanalytical capabilities go beyond this initial 
remit. This is evidenced by the distinct oxidation peak highlighted in Figure 7(D). As noted in the introduction, carbon has a wide potential window and the expansion of the base system towards the analysis of other electroactive biomarkers is clearly possible and could be a viable alternative to applications presently being characterised with screen printed electrodes [19-21].

\subsection{Conclusions}

Microneedle sensors offer a multitude of advantages over conventional screen printed systems and the composite approach taken could aid the translation of the latter to the newer format. The micro-moulding technique in particular enables the rapid, low cost production of sensors that have wide versatility and while the carbon-polystyrene system detailed has been used for $\mathrm{pH}$ measurements, it is clear from electrochemical characterisation conducted that the system has a wide potential range through which numerous analytes could be detected. While the composite $\mathrm{MN}$ patches have been evaluated with tomato skin, the piercing capability has been proven and augers well for translation to biomedical contexts.

\section{Acknowledgements}

The authors are pleased to acknowledge financial support from the European Union's INTERREG VA Programme, managed by the Special EU Programmes Body (SEUPB) and the Department for the Economy (DfE) Northern Ireland,

\section{Author Contributions:}

Catherine Hegarty: Design, manufacture and electrochemical characterisation of microneedles Aaron McConville: Optimisation of electron microscopy/CT Scanning Ruairi J McGlynn, Davide Mariotti: Raman spectroscopy investigation, analysis and interpretation James Davis: Supervision of overall project and compilation of manuscript 


\section{References}

1. H. Quinn, M-C. Kearney, A. Courtenay, et al. The role of microneedles for drug and vaccine delivery. Expert Opinion on Drug Delivery, 11 (2014) 1769-1780.

2. K. Cheung, D.B. Das, Microneedles for drug delivery: trends and progress. Drug Delivery, 23 (2016) 2338-2354.

3. N.S. Rejinold, J-H. Shin, H.Y. Seok, et al. Biomedical applications of microneedles in therapeutics: recent advancements and implications in drug delivery. Expert Opinion on Drug Delivery, 13 (2016) 109-131.

4. A. Martin, A. McConville, A. Anderson, A. McLister, J. Davis, Microneedle Manufacture: Assessing Hazards and Control Measures. Safety, 3 (2017) 25

5. A. McConville, J. Davis, Transdermal microneedle sensor arrays based on palladium: Polymer composites. Electrochemistry Communications, 72 (2016) 162-165.

6. T.M. Rawson, S. Sharma, P. Georgiou, A. Holmes, A.E.G. Cass, D. O'Hare, Towards a minimally invasive device for beta-lactam monitoring in humans, Electrochemistry Communications 82 (2017) 1-5

7. S. Sharma, Z. Huang, M. Rogers, M. Boutelle, A.E.G. Cass, Evaluation of a minimally invasive glucose biosensor for continuous tissue monitoring, Anal Bioanal Chem 408 (2016) 8427-8435

8. S. Sharma, A. El-Laboudi, M. Reddy, N. Jugnee, S. Sivasubramaniyam, M. El Sharkawy, M (EI Sharkawy, P. Georgiou, D. Johnston, N. Oliver, A.E.G. Cass, A pilot study in humans of microneedle sensor arrays for continuous glucose monitoring, Analytical Methods, 10 (2018) 2088-2095

9. P.P. Samant, M. R. Prausnitz, Mechanisms of sampling interstitial fluid from skin using a microneedle patch, PNAS 115 (2018) 4583-4588

10. H. Chang, M. Zheng, X. Yu, A. Than, R. Z. Seeni, R. Kang, J. Tian, D. P. Khanh, L. Liu, P. Chen, C. $\mathrm{Xu}$, A Swellable Microneedle Patch to Rapidly Extract Skin Interstitial Fluid for Timely Metabolic Analysis, Adv. Mater. 29 (2017) 1702243

11. B. Q. Tran, P.R. Miller, R.M. Taylor, G. Boyd, P.M. Mach, C. N. Rosenzweig,J.T. Baca, R. Polsky, T. Glaros, Proteomic Characterization of Dermal Interstitial Fluid Extracted Using a Novel Microneedle-Assisted Technique, J.Proteome Res. 17 (2018) 479-485

12. S.R. Chinnadayyala, K. D. Park, S. Cho, Review-In Vivo and In Vitro Microneedle Based Enzymatic and Non-Enzymatic Continuous Glucose Monitoring Biosensors, ECS Journal of Solid State Science and Technology, 7 (2018) Q3159-Q3171

13. S. Anastasova, B. Crewther, P. Bembnowicz, V. Curto, H.M.D. Ipa, B.Rosa, G-Z. Yanga, A wearable multi sensing patch for continuous sweat monitoring, Biosensors and Bioelectronics 93 (2017) 139-145

14. S.R. Chinnadayyala, I.Park, S. Cho, Non enzymatic determination of glucose at near neutral pH values based on the use of nafion and platinum black coated microneedle electrode array, Microchimica Acta 185 (2018) 250

15. B. Ciui, A. Martin, R.K. Mishra, B. Brunetti, T. Nakagawa, T.J. Dawkins, M.J. Lyu, C.Cristea, R. Sandulescu, J. Wang, Wearable Wireless Tyrosinase Bandage and Microneedle Sensors: 
Toward Melanoma Screening, Advanced Healthcare Materials, 7 (2018) Article Number: 1701264

16. J.X. Zhou, L.N. Tang, F. Yang, F.X. Liang, H. Wang, Y.T. Li, G.J. Zhang, MoS2/Pt nanocompositefunctionalized micro-needle for real-time monitoring of hydrogen peroxide release from living cells, Analyst, 142 (2017) 4322-4329

17. T. M. Rawson, D. O'Hare, P. Herrero, S. Sharma, L. S. P. Moore, E. de Barra, J. A. Roberts, A. C. Gordon, W. Hope, P. Georgiou, A. E. G. Cass and A. H. Holmes, Delivering precision antimicrobial therapy through closed-loop control systems, J Antimicrob Chemother 73 (2018) 835-843

18. N.V. Apollo, M.I. Maturana, W.Tong, D.A.X. Nayagam, M.N. Shivdasani, J.Foroughi , G.G. Wallace, S. Prawer, M.R. Ibbotson, D.J. Garrett, Soft, Flexible Freestanding Neural Stimulation and Recording Electrodes Fabricated from Reduced Graphene Oxide, Adv. Funct. Mater. 25 (2015) 3551-3559

19. M. Li, D.W. Li, G.L. Xiu, Y.T. Long, Applications of screen-printed electrodes in current environmental analysis, Current Opinion in Electrochemistry, 3 (2017) 137-143

20. M. Trojanowicz, Impact of nanotechnology on design of advanced screen-printed electrodes for different analytical applications, TRAC-Trends in Analytical Chemistry,84 (2016) 22-47

21. F. Arduini, L. Micheli, D. Moscone, G. Palleschi, S. Piermarini, F. Ricci, G. Volpe, Electrochemical biosensors based on nanomodified screen-printed electrodes: Recent applications in clinical analysis, TRAC-Trends in Analytical Chemistry, 79 (2016) 114-126

22. E. Chiavazza, S. Berto, A. Giacomino, M. Malandrino, Claudia Barolo, E. Prenesti, D. Vione, O. Abollino, Electrocatalysis in the oxidation of acetaminophen with an electrochemically activated glassy carbon electrode, Electrochimica Acta, 192 (2016) 139-147

23. A. Anderson, J. Phair, J. Benson, B. Meenan, J. Davis, Investigating the use of endogenous quinoid moieties on carbon fibre as means of developing micro $\mathrm{pH}$ sensors, Materials Science and Engineering C, 43 (2014) 533-537

24. McCreery K. K. Cline, M.T. McDermott,R. L. McCreery, Anomalously Slow Electron Transfer at Ordered Graphite Electrodes: Influence of Electronic Factors and Reactive Sites, J. Phys. Chem. 98 (1994) 5314-5319

25. J.J. Hickman, D. Ofer, P.E. Laibinis, G.M.Whitesides,M.S.Wrighton, Molecular self assembly of 2- terminal voltammetric microsensors with internal references, Science 252 (1991) 688-691.

26. V.G.H. Lafitte, W.X. Wang, A.S. Yashina, N.S. Lawrence, Anthraquinone-ferrocene film electrodes: utility in pH and oxygen sensing, Electrochem. Commun. 12 (2008) 1831-1834.

27. A.E. Musa,M.A. Alonso-Lomillo, F.J. del Campo, N. Abramova, O. Dominguez-Renedo, M.J. Arcos-Martinez, J.P. Kutter, Thick-film voltammetric $\mathrm{pH}$-sensors with internal indicator and reference species, Talanta 99 (2012) 737-743.

28. L. Xiong, C. Batchelor-McAuley, R.G. Compton, Calibrationless pH sensors based on nitrosophenyl and ferrocenyl co-modified screen printed electrodes, Sensors Actuators B 159 (2011) 251-255.

29. C. Hegarty, S. Kirkwood, M.F. Cardosi, C.L. Lawrence, C.M. Taylor, R.B. Smith, J. Davis, Disposable solid state $\mathrm{pH}$ sensor based on flavin polymer-ferrocyanide redox couples. Microchemical Journal, 139 (2018) 210-215 
30. N.S. Lawrence, E.L. Beckett, J. Davis, R.G. Compton, Advances in the voltammetric analysis of small biologically relevant compounds, Analytical Biochemistry, 303 (2002)1-16

31. S. Baek, E. Jeon, K. S. Park, K-H. Yeo, and J. Lee, Monitoring of Water Transportation in Plant Stem With Microneedle Sap Flow Sensor, J. Microelectromechanical Systems 27 (2018) 440447

32. E. Jeon, S. Choi, K-H. Yeo, K.S. Park, M. L. Rathod, J. Lee, Development of electrical conductivity measurement technology for key plant physiological information using microneedle sensor, J. Micromech. Microeng. 27 (2017) 085009 